VOL. 22 (1980), 407-409.

\title{
THE ADDITIVE GROUPS OF SUBDIRECTLY IRREDUCIBLE RINGS II
}

\author{
ShALOM FEIGELSTOCK
}

\begin{abstract}
The classification of strongly subdirectly irreducible rings, which was begun in a previous paper, is completed by showing that a mixed group $G$ is strongly subdirectly irreducible if and only if $G \simeq Z\left(p^{\infty}\right) \oplus H, H$ a rank one, $p$-divisible, torsion free nil group.
\end{abstract}

I.

In [1] the strongly subdirectly irreducible torsion, and torsion free groups were classified. The following necessary condition was obtained for a mixed group to be strongly subdirectly irreducible [1, Theorem 3.3].

THEOREM A. Let $G$ be a mixed strongly subdirectly irreducible group. Then $G \simeq Z\left(p^{\infty}\right) \oplus H, H$ a rank one, torsion free nil group.

The object of this short note is to give necessary and sufficient conditions for a mixed group to be strongly subdirectly irreducible, and so complete the classification of the strongly subdirectly irreducible groups.

II.

THEOREM B. Let $G$ be a mixed group. $G$ is strongly subdirectly irreducible if and only if $G \simeq Z\left(p^{\infty}\right) \oplus H, H$ a rank one, p-divisible, torsion free nil group.

Proof. Suppose that $G$ is strongly subdirectly irreducible. By

Received 13 June 1980. 
Theorem $A, G \simeq Z\left(p^{\infty}\right) \oplus H, H$ a rank one, torsion free, nil group. It suffices to show that $H$ is $p$-divisible. If not, there exists $h \in H$, $h \neq 0$, such that the $p$-height of $h$ is 0 . For $a, b \in G$ define $a \cdot b=0$ if $a \in Z\left(p^{\infty}\right)$ or $b \in Z\left(p^{\infty}\right)$. Choose $a_{0} \in Z\left(p^{\infty}\right)$ with $\left|a_{0}\right|=p$. For $h_{1}, h_{2} \in H$, there exist positive integers $n_{i}$, and integers $m_{i}$ such that $p / n_{i}$, and $n_{i} h_{i}=m_{i} h, i=1,2$. There exists a unique element $a \in Z\left(p^{\infty}\right)$ such that $n_{1} n_{2} a=m_{1} m_{2} a_{0}$. Define $h_{1} h_{2}=a$. The above products define a ring structure $R$ on $G$, with ideals $Z\left(p^{\infty}\right)$ and $p H$. Clearly $Z\left(p^{\infty}\right) \cap p H=0$, and $h^{2}=a_{0} \neq 0$. Hence $R$ is a ring satisfying $R^{2} \neq 0$, but $R$ is not subdirectly irreducible, a contradiction.

Let $G \simeq Z\left(p^{\infty}\right) \oplus H, H$ a rank one, $p$-divisible, torsion free nil group. Clearly $G$ is p-divisible. $G$ is not nil [2, Theorem 120.3]. Let $R$ be a ring with $R^{+}=G$, and $R^{2} \neq 0$. The quotient ring $R / Z\left(p^{\infty}\right)$ has a nil additive group. Hence $R^{2} \subseteq Z\left(p^{\infty}\right)$. Let $a_{0} \in Z\left(p^{\infty}\right)$, $\left|a_{0}\right|=p$. Every non-zero subgroup of $Z\left(p^{\infty}\right)$ contains $a_{0}$. Let $I$ be an ideal in $R, I \neq 0$. Suppose that $a_{0} \& I$. Then $I \cap Z\left(p^{\infty}\right)=0$. However $R I \subseteq R^{2} \subseteq Z\left(p^{\infty}\right)$, and so $R I=0$, and similarly $I R=0$. Let $0 \neq x \in I, \quad x=a+h, \quad a \in Z(p), \quad h \in H$. Clearly $h \neq 0$. There exists a positive integer $n$ such that $p^{n} a=0$. Hence $p^{n} x \in H \cap I$. We may therefore assume that there exists $0 \neq h_{0} \in H \cap I$. Let $h \in H$. There exist a non-negative integer $k$, a positive integer $r$, and an integer $s$ such that $p / r$, and $p^{k} r h=s h_{0}$. Therefore $p^{k} r h \subseteq I R=0$. However $p^{k} r h R=r h\left(p^{k} R\right)=r h R$, and so $r h R=0$. Now $h R \subseteq Z\left(p^{\infty}\right)$, and for $0 \neq a \in Z\left(p^{\infty}\right), \quad r a \neq 0$. Hence $h R=0$. Therefore

(1) $H R=0$, and similarly

(2) $R H=0$.

Let $a \in Z\left(p^{\infty}\right), x \in R$. There exists a positive integer $n$ such that $p^{n} a=0$, and there exists $y \in R$ such that $x=p^{n} y$. Hence 
$a \cdot x=a \cdot\left(p^{n} y\right)=\left(p^{n} a\right) \cdot y=0$. Therefore

(3) $Z\left(p^{\infty}\right) \cdot R=0$, and similarly

(4) $R \cdot Z\left(p^{\infty}\right)=0$.

Equalities (1), (2), (3), and (4), imply that $R^{2}=0$, a contradiction.

\section{References}

[1] Shalom Feigelstock, "The additive groups of subdirectly irreducible rings", Bull. Austral. Math. Soc. 20 (1979), 165-170.

[2] László Fuchs, Infinite abelian groups, Volume II (Pure and Applied Mathematics, 36-II. Academic Press, New York and London, 1973).

Department of Mathematics,

Bar-l Ian University,

Ramat-Gan,

Israel. 